\title{
QCloud2: An Improved Cloud-based Quality-Control System for Mass-Spectrometry-based Proteomics Laboratories
}

\author{
Roger Olivella, ${ }^{\S}$ Cristina Chiva, ${ }^{\S}$ Marc Serret, Daniel Mancera, Luca Cozzuto, Antoni Hermoso, \\ Eva Borràs, Guadalupe Espadas, Julia Morales, Olga Pastor, Amanda Solé, Julia Ponomarenko, \\ and Eduard Sabidó*
}

Cite This: J. Proteome Res. 2021, 20, 2010-2013

Read Online

ACCESS | 네 Metrics \& More | 回 Article Recommendations | sl Supporting Information

ABSTRACT: QCloud is a cloud-based system to support proteomics laboratories in daily quality assessment using a userfriendly interface, easy setup, and automated data processing. Since its release, QCloud has facilitated automated quality control for proteomics experiments in many laboratories. QCloud provides a quick and effortless evaluation of instrument performance that helps to overcome many analytical challenges derived from clinical and translational research. Here we present an improved version of the system, QCloud2. This new version includes enhancements in the scalability and reproducibility of the quality-control pipelines, and it

\section{QCloud2}
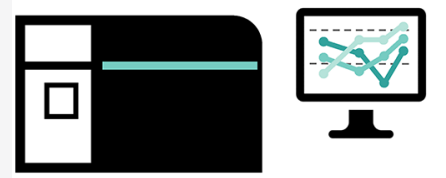

quality control
User Management \& Roles

Guidesets \& Thresholds

Instrument Systems (LC-MS)

Annotation System (Actions \& Problems) features an improved front end for data visualization, user management, and chart annotation. The QCloud2 system also includes programmatic access and a standalone local version.

KEYWORDS: quality control, proteomics, cloud computing, computational proteomics

\section{INTRODUCTION}

In 2018, our laboratory introduced QCloud, a cloud-based system to support proteomics laboratories in daily quality assessment using a user-friendly interface, easy setup, and automated data processing. ${ }^{1}$ Since its release, QCloud has facilitated automated quality control (QC) for proteomics experiments within the European alliance Core for Life (https://coreforlife.eu $)^{2,3}$ and many other proteomics laboratories across the world ( 22 active laboratories within the last 6 months, 133 users, and 59 instruments). ${ }^{4,5}$ QCloud provides a quick and effortless evaluation of instrument performance that helps to overcome many analytical challenges derived from clinical and translational research. Here we present an improved version of the system, QCloud2. This new version includes enhancements in the scalability and reproducibility of the QC pipelines, and it features an improved front end for data visualization, user management, and chart annotation. The QCloud2 system also includes programmatic access and a standalone local version. The new version is accessible at https://qcloud2.crg.eu, and its source code is open to the proteomics community (https://github.com/ proteomicsunitcrg/qcloud2-pipeline).

\section{ENHANCEMENT OF SCALABILITY AND REPRODUCIBILITY}

The QCloud2 system comprises a thin client installed in the mass spectrometer acquisition computer, a cloud-based processing pipeline, and a web application that together automate the complete QC workflow.

In QCloud2, the processing pipeline has been scaled to support dozens of instruments and sites by developing a fully automated setup, ensuring scalability and ease of deployment, in alignment with current reusability principles. ${ }^{6,7}$ In QCloud2, the automated QC pipelines are based on the OpenMS ${ }^{8}$ modules, but the workflow structures have been moved to $\mathrm{KNIME}^{9}$ and Nextflow ${ }^{10}$ to ensure reproducibility of the QC analysis and easy adoption within the community (Figure 1A). Altogether, these changes allowed us to automate most development requirements of the pipeline, including parallel computing, easy high-performance computing (HPC) cluster deployment, and central processing unit (CPU) and memory management. Moreover, the pipeline incorporates the ThermoRawFileReader library ${ }^{11}$ that enables direct access to raw data from Thermo mass spectrometers in Linux environments.

Special Issue: Software Tools and Resources 2021

Received: October 29, 2020

Published: March 16, 2021 


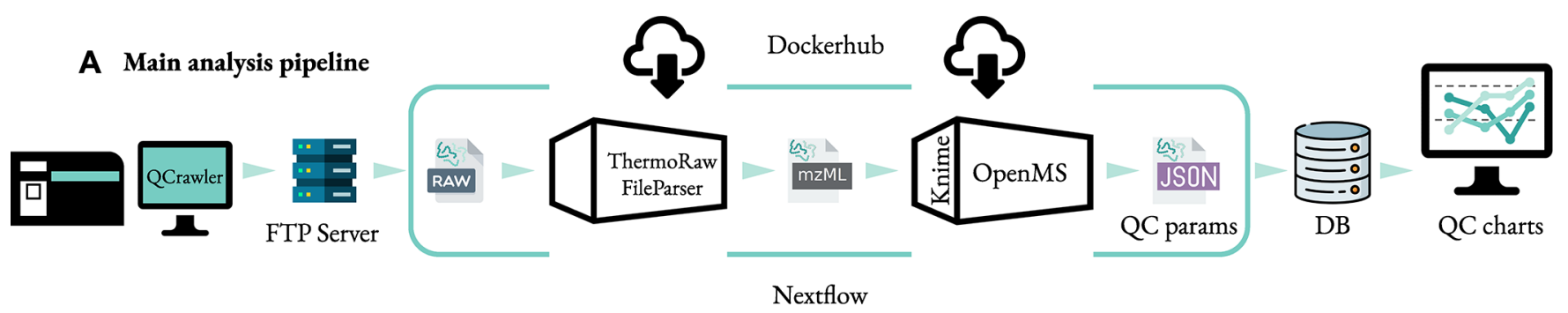

\section{B New front-end features}
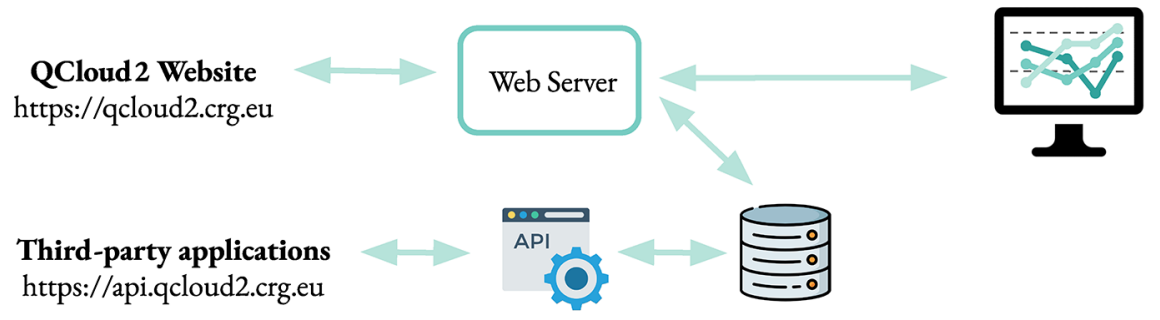

User Management \& Roles

https://api.qcloud2.crg.eu

Guidesets \& Thresholds

Instrument Systems (LC-MS)

Annotation System

(Actions \& Problems)

Figure 1. Schematic representation of the main analysis pipeline (A) and new front-end features of QCloud2 (B).

The source code of the new QCloud2 QC pipeline is available at https://github.com/proteomicsunitcrg/qcloud2pipeline.

\section{IMPROVED DATA VISUALIZATION, USER MANAGEMENT, EVALUATION, AND ANNOTATION SYSTEMS}

The QCloud2 web application was redesigned with Spring Boot, ${ }^{12}$ the Angular ${ }^{13}$ framework, and the Plotly.js ${ }^{14}$ library for enhanced performance, and the visualization of the QC data was improved (Figure 1B). We expanded the data visualization options and enabled the development of new customized views. In contrast with the fixed selection of charts in the previous version of QCloud, new customized views allow users to set as default any combination of QC metrics charts available in QCloud2.

Other new features within the QCloud2 web application include (i) user management, (ii) setting of guidesets and thresholds, (iii) registration of new instruments, and (iv) troubleshooting annotations. Thus the new user management feature allows users to be organized in different groups (e.g., by laboratories) and different roles (User, Lab Manager, and Administrator) (Figure S1). New remodeled guidesets and thresholds now allow users to flexibly set and manage periods of good instrument performance for a particular sample type (Figures S2 and S3). These periods are defined as reference guidesets, and their variance is then used to compute the thresholds that are applied to the QC parameters. With these thresholds, QCloud2 automatically classifies each data point as being conforming or nonconforming.

We also introduced a module to allow users to define and register different combinations of liquid chromatography and mass spectrometers as instrument entities in their laboratories (Figures S4-S6). Within the definition of the laboratory LCMS systems, QCloud2 has extended its support to the Human Proteome Organization (HUPO)-Proteomics Standards Initiative (PSI) mass spectrometry controlled vocabulary, ${ }^{15}$ and at the same time, it has incorporated new terms to include liquid chromatography instruments (https://github.com/ proteomicsunitcrg/cv/blob/master/lc-cv.obo) and additional QC parameters (https://github.com/proteomicsunitcrg/cv/ $\mathrm{blob} / \mathrm{master}$ /qc-cv.obo). New controlled vocabulary has also been introduced for the new troubleshooting annotation features. This controlled vocabulary is divided in two main categories, problems and actions, and each of has a hierarchical set of terms to cover different events in the LC-MS instruments (https://github.com/proteomicsunitcrg/cv/blob/ master/ts-cv.obo). The annotation system allows users to annotate specific data points with specific problems or actions by directly selecting one or more terms in the QCloud2 web interface (Figure S7).

The new QCloud2 version is accessible at https://qcloud2. crg.eu and a demonstration version is available at https:// demo.qcloud2.crg.eu.

\section{PROGRAMMATIC ACCESS AND STANDALONE LOCAL VERSION}

Finally, a standalone local version of QCloud2 and a RESTful API for programmatic access to QCloud2 data have been developed. The new RESTful API (https://documenter. getpostman.com/view/8947947/SVn3qu8K) allows users to use third-party applications to have read access to their own QC data, and all of its technical documentation is found at https://documenter.getpostman.com/view/8947947/ SVn3qu8K.

The standalone local version of QCloud2 (or QLV, which stands for QCloud2 Local Version) was developed to allow its installation on HPC clusters and local servers, including both the data analysis pipeline and the web application. The detailed documentation for the QLV is found at https://github.com/ proteomicsunitcrg/qcloud2-pipeline/tree/local.

\section{CONCLUSIONS}

A new version of the QCloud system was developed with enhanced scalability and reproducibility of the QC pipelines, and it features an improved front end for data visualization, 
user management, and chart annotation. The updated QCloud2 allows full reproducibility of the analysis pipelines, facilitates the adoption within the community, and increases its throughput of processing data from multiple instruments and laboratories.

\section{ASSOCIATED CONTENT}

\section{SI Supporting Information}

The Supporting Information is available free of charge at https://pubs.acs.org/doi/10.1021/acs.jproteome.0c00853.

Supplementary Figure S1. New user management features. Supplementary Figure S2. Graphical user interface to set the parameters for thresholds. Supplementary Figure S3. Graphical user interface to set and manage guidesets. Supplementary Figure S4. New instrument management feature to register new LC systems. Supplementary Figure S5. New instrument management feature to register new MS systems. Supplementary Figure S6. Instrument management feature to set laboratory LC-MS systems. Supplementary Figure S7. New annotation system to specify problems and actions (PDF)

\section{AUTHOR INFORMATION}

\section{Corresponding Author}

Eduard Sabidó - Centre de Regulació Genòmica (CRG), Barcelona Institute of Science and Technology (BIST), Barcelona 08003, Spain; Universitat Pompeu Fabra (UPF), Barcelona 08003, Spain; 이이이.org/0000-0001-65067714; Email: eduard.sabido@crg.cat

Authors

Roger Olivella - Centre de Regulació Genòmica ( $C R G$ ), Barcelona Institute of Science and Technology (BIST), Barcelona 08003, Spain; Universitat Pompeu Fabra (UPF), Barcelona 08003, Spain

Cristina Chiva - Centre de Regulació Genòmica (CRG), Barcelona Institute of Science and Technology (BIST), Barcelona 08003, Spain; Universitat Pompeu Fabra (UPF), Barcelona 08003, Spain

Marc Serret - Centre de Regulació Genòmica (CRG), Barcelona Institute of Science and Technology (BIST), Barcelona 08003, Spain; Universitat Pompeu Fabra (UPF), Barcelona 08003, Spain

Daniel Mancera - Centre de Regulació Genòmica ( $C R G$ ), Barcelona Institute of Science and Technology (BIST), Barcelona 08003, Spain; Universitat Pompeu Fabra (UPF), Barcelona 08003, Spain

Luca Cozzuto - Centre de Regulació Genòmica (CRG), Barcelona Institute of Science and Technology (BIST), Barcelona 08003, Spain

Antoni Hermoso - Centre de Regulació Genòmica (CRG), Barcelona Institute of Science and Technology (BIST), Barcelona 08003, Spain

Eva Borràs - Centre de Regulació Genòmica (CRG), Barcelona Institute of Science and Technology (BIST), Barcelona 08003, Spain; Universitat Pompeu Fabra (UPF), Barcelona 08003, Spain

Guadalupe Espadas - Centre de Regulació Genòmica ( $C R G)$, Barcelona Institute of Science and Technology (BIST), Barcelona 08003, Spain; Universitat Pompeu Fabra (UPF), Barcelona 08003, Spain
Julia Morales - Centre de Regulació Genòmica ( $C R G$ ), Barcelona Institute of Science and Technology (BIST), Barcelona 08003, Spain; Universitat Pompeu Fabra (UPF), Barcelona 08003, Spain

Olga Pastor - Centre de Regulació Genòmica (CRG), Barcelona Institute of Science and Technology (BIST), Barcelona 08003, Spain; Universitat Pompeu Fabra (UPF), Barcelona 08003, Spain

Amanda Solé - Centre de Regulació Genòmica (CRG), Barcelona Institute of Science and Technology (BIST), Barcelona 08003, Spain; Universitat Pompeu Fabra (UPF), Barcelona 08003, Spain

Julia Ponomarenko - Centre de Regulació Genòmica (CRG), Barcelona Institute of Science and Technology (BIST), Barcelona 08003, Spain; Universitat Pompeu Fabra (UPF), Barcelona 08003, Spain

Complete contact information is available at:

https://pubs.acs.org/10.1021/acs.jproteome.0c00853

\section{Author Contributions}

${ }^{\S}$ R.O. and C.C. contributed equally.

Notes

The authors declare no competing financial interest.

\section{ACKNOWLEDGMENTS}

The CRG/UPF Proteomics Unit is part of the Spanish Infrastructure for Omics Technologies (ICTS OmicsTech), and it is a member of the ProteoRed PRB3 consortium, which is supported by grant PT17/0019 of the PE I+D+i 2013-2016 from the Instituto de Salud Carlos III (ISCIII), ERDF, and "Secretaria d'Universitats i Recerca del Departament d'Economia i Coneixement de la Generalitat de Catalunya" (2017SGR595). This project was supported by EPIC-XS, project number 823839, funded by the Horizon 2020 Programme of the European Union. We also acknowledge support of the Spanish Ministry of Science and Innovation to the EMBL partnership, the Centro de Excelencia Severo Ochoa and the CERCA Programme from Generalitat de Catalunya.

\section{REFERENCES}

(1) Chiva, C.; Olivella, R.; Borràs, E.; Espadas, G.; Pastor, O.; Solé, A.; Sabidó, E. QCloud: A Cloud-Based Quality Control System for Mass Spectrometry-Based Proteomics Laboratories. PLoS One 2018, 13 (1), No. e0189209.

(2) Meder, D.; Morales, M.; Pepperkok, R.; Schlapbach, R.; Tiran, A.; Van Minnebruggen, G. Institutional Core Facilities: Prerequisite for Breakthroughs in the Life Sciences: Core Facilities Play an Increasingly Important Role in Biomedical Research by Providing Scientists Access to Sophisticated Technology and Expertise. EMBO Rep. 2016, 17 (8), 1088-1093.

(3) Meder, D.; Van Minnebruggen, G. Straight Talk with...Doris Meder and Geert Van Minnebruggen. Interview by Katharine Sanderson. Nat. Med. 2013, 19 (7), 802.

(4) Gordon, D. E.; Jang, G. M.; Bouhaddou, M.; Xu, J.; Obernier, K.; White, K. M.; O’Meara, M. J.; Rezelj, V. V.; Guo, J. Z.; Swaney, D. L.; et al. A SARS-CoV-2 Protein Interaction Map Reveals Targets for Drug Repurposing. Nature 2020, 583, 459-468.

(5) Maia, T. M.; Staes, A.; Plasman, K.; Pauwels, J.; Boucher, K.; Argentini, A.; Martens, L.; Montoye, T.; Gevaert, K.; Impens, F. Simple Peptide Quantification Approach for MS-Based Proteomics Quality Control. ACS Omega 2020, 5 (12), 6754-6762.

(6) Wilkinson, M. D.; Dumontier, M.; Aalbersberg, Ij. J.; Appleton, G.; Axton, M.; Baak, A.; Blomberg, N.; Boiten, J.-W.; da Silva Santos, 
L. B.; Bourne, P. E.; Bouwman, J.; Brookes, A. J.; Clark, T.; Crosas, M.; Dillo, I.; Dumon, O.; Edmunds, S.; Evelo, C. T.; Finkers, R.; Gonzalez-Beltran, A.; Gray, A. J. G.; Groth, P.; Goble, C.; Grethe, J. S.; Heringa, J.; 't Hoen, P. A. C.; Hooft, R.; Kuhn, T.; Kok, R.; Kok, J.; Lusher, S. J.; Martone, M. E.; Mons, A.; Packer, A. L.; Persson, B.; Rocca-Serra, P.; Roos, M.; van Schaik, R.; Sansone, S.-A.; Schultes, E.; Sengstag, T.; Slater, T.; Strawn, G.; Swertz, M. A.; Thompson, M.; van der Lei, J.; van Mulligen, E.; Velterop, J.; Waagmeester, A.; Wittenburg, P.; Wolstencroft, K.; Zhao, J.; Mons, B. The FAIR Guiding Principles for Scientific Data Management and Stewardship. Sci. Data 2016, 3, 160018.

(7) Jiménez, R. C.; Kuzak, M.; Alhamdoosh, M.; Barker, M.; Batut, B.; Borg, M.; Capella-Gutierrez, S.; Chue Hong, N.; Cook, M.; Corpas, M.; Flannery, M.; Garcia, L.; Gelpí, J. Ll.; Gladman, S.; Goble, C.; González Ferreiro, M.; Gonzalez-Beltran, A.; Griffin, P. C.; Grüning, B.; Hagberg, J.; Holub, P.; Hooft, R.; Ison, J.; Katz, D. S.; Leskošek, B.; López Gómez, F.; Oliveira, L. J.; Mellor, D.; Mosbergen, R.; Mulder, N.; Perez-Riverol, Y.; Pergl, R.; Pichler, H.; Pope, B.; Sanz, F.; Schneider, M. V.; Stodden, V.; Suchecki, R.; SvobodováVařeková, R.; Talvik, H.-A.; Todorov, I.; Treloar, A.; Tyagi, S.; van Gompel, M.; Vaughan, D.; Via, A.; Wang, X.; WatsonHaigh, N. S.; Crouch, S. Four Simple Recommendations to Encourage Best Practices in Research Software. F1000Research 2017, 6, 876.

(8) Röst, H. L.; Sachsenberg, T.; Aiche, S.; Bielow, C.; Weisser, H.; Aicheler, F.; Andreotti, S.; Ehrlich, H.-C.; Gutenbrunner, P.; Kenar, E.; Liang, X.; Nahnsen, S.; Nilse, L.; Pfeuffer, J.; Rosenberger, G.; Rurik, M.; Schmitt, U.; Veit, J.; Walzer, M.; Wojnar, D.; Wolski, W. E.; Schilling, O.; Choudhary, J. S.; Malmström, L.; Aebersold, R.; Reinert, K.; Kohlbacher, O. OpenMS: A Flexible Open-Source Software Platform for Mass Spectrometry Data Analysis. Nat. Methods 2016, 13 (9), 741-748.

(9) Berthold, M. R.; Cebron, N.; Dill, F.; Gabriel, T. R.; Kötter, T.; Meinl, T.; Ohl, P.; Thiel, K.; Wiswedel, B. KNIME-the Konstanz Information Miner: Version 2.0 and Beyond. AcM SIGKDD Explor. Newsl. 2009, 11 (1), 26-31.

(10) Di Tommaso, P.; Chatzou, M.; Floden, E. W.; Barja, P. P.; Palumbo, E.; Notredame, C. Nextflow Enables Reproducible Computational Workflows. Nat. Biotechnol. 2017, 35 (4), 316-319.

(11) Hulstaert, N.; Shofstahl, J.; Sachsenberg, T.; Walzer, M.; Barsnes, H.; Martens, L.; Perez-Riverol, Y. ThermoRawFileParser: Modular, Scalable, and Cross-Platform RAW File Conversion. J. Proteome Res. 2020, 19 (1), 537-542.

(12) Spring. https://spring.io/ (accessed Oct. 25, 2020).

(13) Angular. https://angular.io/ (accessed Oct. 25, 2020).

(14) Plotly: The front end for ML and data science models. https:// plotly.com/ (accessed Oct. 25, 2020).

(15) Mayer, G.; Montecchi-Palazzi, L.; Ovelleiro, D.; Jones, A. R.; Binz, P.-A.; Deutsch, E. W.; Chambers, M.; Kallhardt, M.; Levander, F.; Shofstahl, J. The HUPO Proteomics Standards Initiative-Mass Spectrometry Controlled Vocabulary. Database 2013, 2013. 